\title{
Microencapsulation of thyme oil by coacervation
}

\author{
Isabel M. Martins ${ }^{1}$, Filomena Barreiro ${ }^{2}$, Alírio E. Rodrigues ${ }^{1 *}$ \\ ${ }^{1}$ LSRE - Laboratory of Separation and Reaction Engineering, Department of \\ Chemical Engineering, Faculty of Engineering of University of Porto, Rua Dr \\ Roberto Frias, 4200-465 Porto, Portugal \\ ${ }^{2}$ LSRE - Laboratory of Separation and Reaction Engineering, Bragança Polytechnic \\ Institute, Campus Santa Apolónia Ap 1134, 5301-857 Bragança, Portugal
}

Keywords: Polylactide, thyme oil, coacervation, microencapsulation.

Topic: Sustainable process-product development through green chemistry.

Microencapsulation is a technology that includes several processes to cover an active agent with a protective wall material. There are various industrial applications, such as carbonless paper, "scratch and sniff" fragrance sampling, "intelligent" textiles, controlled release of drugs, pesticides and cosmetics. A wide range of core materials have been encapsulated, including adhesives, agrochemicals, live cells, active enzymes, flavours, fragrances and pharmaceuticals (Gosh, 2006). Microencapsulation techniques can be as diverse as coacervation (Jégat, 2000; Soper et al., 2000), atomization (Ascheri et al., 2003), interfacial polymerisation (Mizuno et al., 2005; Hirech et al., 2003) and "in situ" polymerisation (Bang et al., 2005). The choice of the appropriate technique depends on the core material properties, the involved manufacturing conditions and the product end user requirements.

Complex coacervation was the chosen technique to produce polylactide (PLA) microcapsules using Thymus Vulgaris L. (thyme oil), an antioxidant and antimicrobian agent as the core material. This technique consists in the separation of an aqueous polymeric solution into two immiscible liquid phases: a dense coacervate phase and a dilute equilibrium phase. The dense coacervate phase wraps as an uniform layer around suspended core materials.

Biodegradable microcapsules of PLA have received extensive attention as delivery systems for drug encapsulation. This type of biodegradable polymeric carriers can be hydrolyzed in the body to form products that are easily reabsorbed or eliminated (Huang et al., 1997). The core material, thyme oil, is extracted from an aromatic plant of increasing economic importance in North America, Europe and North Africa, so it has an important and growing place in the world market (Badi et al., 2004). This essential oil is widely used in the flavour and food industries. As a pharmaceutical compound, thymol and carvacrol are used in mouthwashes, soaps and creams. Thyme oil is also used in manufacture of perfumes and cosmetics.

In this work we have developed a process to encapsulate thyme oil using PLA as the wall material. Firstly, an oil-in-water (o/w) emulsion stabilized with tween ${ }^{\circledR} 20$ (HLB of 16,7) and a solution of PLA in dimethylformamide (DMF) have been prepared. Thereafter, the PLA solution was added to the previously prepared o/w emulsion. The $\mathrm{o} / \mathrm{w}$ emulsion was obtained by dispersion with an ultraturrax and the encapsulation process proceeded under stirring using an impeller stirrer in a batch actor. The microcapsules formed were hardened by adding octamethylcyclotetrasiloxane and allowed to stand during one hour. After this step, they were decanted and washed with pluronic ${ }^{\circledR} \mathrm{F} 68$ solution $(0.1 \% \mathrm{w} / \mathrm{w})$, an ethanol solution $(30 \% \mathrm{v} / \mathrm{v})$, and hexane. Finally, the microcapsules were freeze-dried during $24 \mathrm{~h}$.

Microcapsules particle size distributions were determined by laser dispersion using a Coulter LS230. It was observed a bimodal distribution in volume with a mean particle

\footnotetext{
*Corresponding author. Tel + 351-22 508 1671. E-mail: arodrig@fe.up.pt.
} 
size of $40 \mu \mathrm{m}$. Microcapsules analysis by optical microscopy (Leica DM 2000 microscopy equipped with software Leica Application Suite Interactive measurement) and by cryogenic scanning electron microscopy (JEOL JSM-6301F/Inca energy $350 /$ Gatan alto 2500) have confirmed the spherical shape, the rough surface, and allowed to estimate the wall thickness around $5 \mu \mathrm{m}$. Moreover, it was observed two predominant sizes of microcapsules, compatible with a bimodal distribution and the absence of agglomerates. Quantification of the encapsulated thyme oil was performed by gas chromatography GC/FID using the thymol peak area. The chromatogram analysis also allowed to inspect for the quality of the encapsulated oil and have shown that apolar compounds of thyme oil were preferentially encapsulated in detriment of the polar ones.

This work intends to develop a coacervation technique to produce microcapsules of PLA to encapsulate thyme oil that will be used on cosmetics or drugs. Control of size and wall thickness of and encapsulation efficiency will be the subject of future work.

\section{References}

Ascheri, D., Marquez, M., Martucci, E. (2003). Microencapsulação de óleo essencial de laranja: selecção de material de parede. Ciência e Tecnologia Alimentar, 23, 1-6.

Badi, H.N., Yazdani, D., Ali, S.M., Nazari, F. (2004). Effects of spacing and harvesting time on herbage yield and quality/quantity of oil in thyme Thymus vulgaris L.. Industrial crops and products, 19, 231-236.

Bang, I., Sung, J., Choi, J. (2005). Synthesis of microcapsule containing oil phase via in-situ polymerization. Journal of Materials Science, 40, 1031-1033.

Gosh, K. (2006). Functional Coatings and Microencapsulation: A General Perspective. WILEY-VCH Verlag GmbH \& Co. KGaA, Weinheim.

Hirech, K., Payan, S., Carnelle, G., Brujes, L., Legrand, J. (2003). Microencapsulation of an insecticide by interfacial polymerisation. Powder Technology, 130, 324-330.

Huang, Y., Chung, T., Tzeng, T. (1997). Drug release from PLA/PEG microparticulates. International Journal of Pharmaceutics, 156. 9-15.

Jégat, C., Taverdet, J.L. (2000). Stirring speed influence on the microencapsulation process and on the drug release from microcapsules. Polymer bulletin, 44, 345351.

Mizuno, K., Tagushi, Y., Tanaka, M. (2005). The effect of the surfactant adsorption layer on the growth rate of polyurethane capsule shell. Journal of Chemical Enineering of Japan, 38, 45-48.

Soper, J.C., Kimand, Y.D., Thomas, M.T. (2000). Method of encapsulating flavours and fragances by controlled water transport into microcapsules. US Patent 6106875. 